\title{
A importância da computação em nuvem para a indústria 4.0
}

Antonio Carlos Menezes Paz zapmca@@gmail.com Centro Universitário Farias Brito (FBUNI), Fortaleza, CE, Brasil

Mauricio Johnny Loos mauricioloos@hotmail.com Centro Universitário Farias Brito (FBUNI), Fortaleza, CE, Brasil

\begin{abstract}
RESUMO
A busca por melhores índices de produtividade e qualidade é cada vez maior, visto que as empresas buscam achar alternativas para serem mais competitivas e sobreviverem no mundo globalizado. Neste contexto este artigo se propõe a apresentar a importância da computação em nuvem para a Indústria 4.0. A indústria atualmente passa por uma transformação visando a digitalização das suas plantas fabris, além da implantação dos conceitos inteligentes nos processos de fabricação. A computação em nuvem, considerada um dos pilares da chamada quarta revolução industrial, auxilia diretamente na digitalização da produção conseguindo criar novos modelos de negócios com base nos dados disponibilizados na nuvem. Com estas informações os usuários poderão prever o encerramento de algumas linhas de produção, programar remotamente linhas complementares, além de efetuarem os seus ajustes em tempo real. A utilização da computação em nuvem possibilita o acesso a recursos de computação praticamente ilimitados, provendo serviços baseados na internet das coisas que permitem a interligação de equipamentos industriais e robôs, através de sensores sofisticados que geram centenas de milhares de informações por segundo, estas informações armazenadas e processadas com extrema velocidade, permite que se atinja novos patamares de eficiência, produtividade e qualidade. Por meio deste estudo pretende-se mostrar como a computação em nuvem é de fundamental importância para indústria 4.0, pois com a utilização correta de suas ferramentas as unidades fabris tornam-se mais ágeis e adaptáveis às variações da produção, evitando perdas e retrabalho.
\end{abstract}

PALAVRAS-CHAVE: Computação em Nuvem, Indústria 4.0, loT. 


\section{INTRODUÇÃO}

De acordo com Wahlster (2012), desde a primeira Revolução Industrial, as revoluções subsequentes resultaram em mudanças radicais na fabricação, da água a máquinas a vapor utilizadas para produzir eletricidade a produção digital automatizada.

Os processos de fabricação tornaram-se cada vez mais complicados, automáticos, o que significa que as pessoas podem operar máquinas de forma simples, eficiente e persistente.

As três primeiras revoluções industriais surgiram como resultado da mecanização, eletricidade e Tecnologia da Informação (TI).

Agora, a Computação em Nuvem (Cloud Computing), aplicada ao ambiente de fabricação, está iniciando uma quarta revolução Industrial.

Inúmeras empresas estão estabelecendo redes globais que incorporam nas suas máquinas, nos seus sistemas de armazenagem e nas suas instalações de produção, o conceito de Cyber-Physical Systems (CPS) e para que isto ocorra de forma rápida e eficiente eles utilizam os recursos de Cloud Computing. Segundo Lee et al. (2014, p.1):

O termo Cyber-Physical Systems (CPS), refere-se a uma nova geração de sistemas com Integração de capacidades computacionais e físicas que podem interagir com os seres humanos através de muitas novas modalidades.

Os sistemas compreendem máquinas inteligentes, sistemas de armazenamento e instalações de produção que possuem a capacidade, de forma autônoma, de trocar informações, desencadeando ações e controle que se relacionam de forma independente. Isto possibilita melhorias nos processos industriais envolvidos na fabricação.

A Indústria 4.0 procura, essencialmente, transformar a própria instalação em um computador ou Fábrica Inteligente (Smart Factories).

Dentro de cada unidade fabril, os processos de fabricação modular podem ser desenvolvidos por meio de sistemas CPS, que incorporam tecnologia de informática nas próprias máquinas, e não apenas nos sistemas para controle remoto e monitoramento.

As fábricas inteligentes já estão começando a empregar uma abordagem completamente nova na sua linha de produção, os produtos são identificáveis de forma exclusiva e podem ser rastreados a qualquer momento em qualquer ponto.

Os sistemas produtivos utilizados por estas unidades fabris são interligados com os fornecedores, desta forma as demandas de insumos a serem utilizados na produção são gerenciadas em tempo real.

A Indústria 4.0 possui um enorme potencial e as Smart Factories permitem a criação de requisitos individuais para o cliente que podem ser formulados lucrativamente ainda que de forma singular. 
A Indústria 4.0 pode nos levar a uma harmonização heterogênea de arquitetura dos sistemas legados, onde diferentes tipos de sistemas conversam e se integram a outras centenas de aplicações.

Isso reduz custos de operação, iniciando investimentos pendentes e instalando conceitos de longo prazo, neste sentido, também se verificam o aumento da segurança em processos de gestão de riscos, com mais transparência e estabilidade.

Dentro da Indústria 4.0, os processos vão permitir mudanças de última hora na produção, além de oferecer a capacidade de responder de forma flexível a interrupções e falhas em toda a linha de produção.

Sendo assim, poderão de forma autônoma permitir a descentralização e automação das decisões em relação à produção, bem como se comunicar e, cooperar via Internet of Things (IoT), com operadores humanos e outras unidades inteligentes, no intuito de completar uma mudança de oferta vertical ou horizontal completa.

Quando inovações tecnológicas começam a ser adotadas em larga escala, discutidas e pesquisadas nos diversos meios, é natural que haja uma busca por uma padronização, iniciando em conceitos de projetos e aplicações, adoções de boas práticas e finalizando em norma, tanto local quanto de abrangência geral e global.

De acordo com Venturelli (2017) quando inovações tecnológicas começam a ser adotadas em larga escala, discutidas e pesquisadas nos diversos meios é natural que haja uma busca por uma padronização.

Adota-se o modelo de referência RAMI.4.0, busca-se mostrar que o modelo utiliza em suas camadas um conjunto de aplicações de soluções de conectividade para projetos aderentes a Indústria 4.0, permitindo um ecossistema cibernético de toda cadeia produtiva.

Destarte, o presente artigo tem por escopo demonstrar como a Computação em Nuvem é importante para a implantação dos conceitos da Indústria 4.0.

Para cumprir seus objetivos, o trabalho primeiramente estabelece o referencial teórico, seguido pelos procedimentos metodológicos adotados, resultados empíricos e, finalmente, suas conclusões.

\section{REVISÃO DA LITERATURA}

\section{DEFINIÇÃO DE CLOUD COMPUTING}

O Instituto Nacional Americano de Padrões e Tecnologia (NIST), define um modelo de Cloud Computing a partir de cinco características essenciais, três modelos de serviço e quatro modelos de implementação, conforme figura 1 . 
Figura 1-Estrutura NIST sobre Cloud Computing

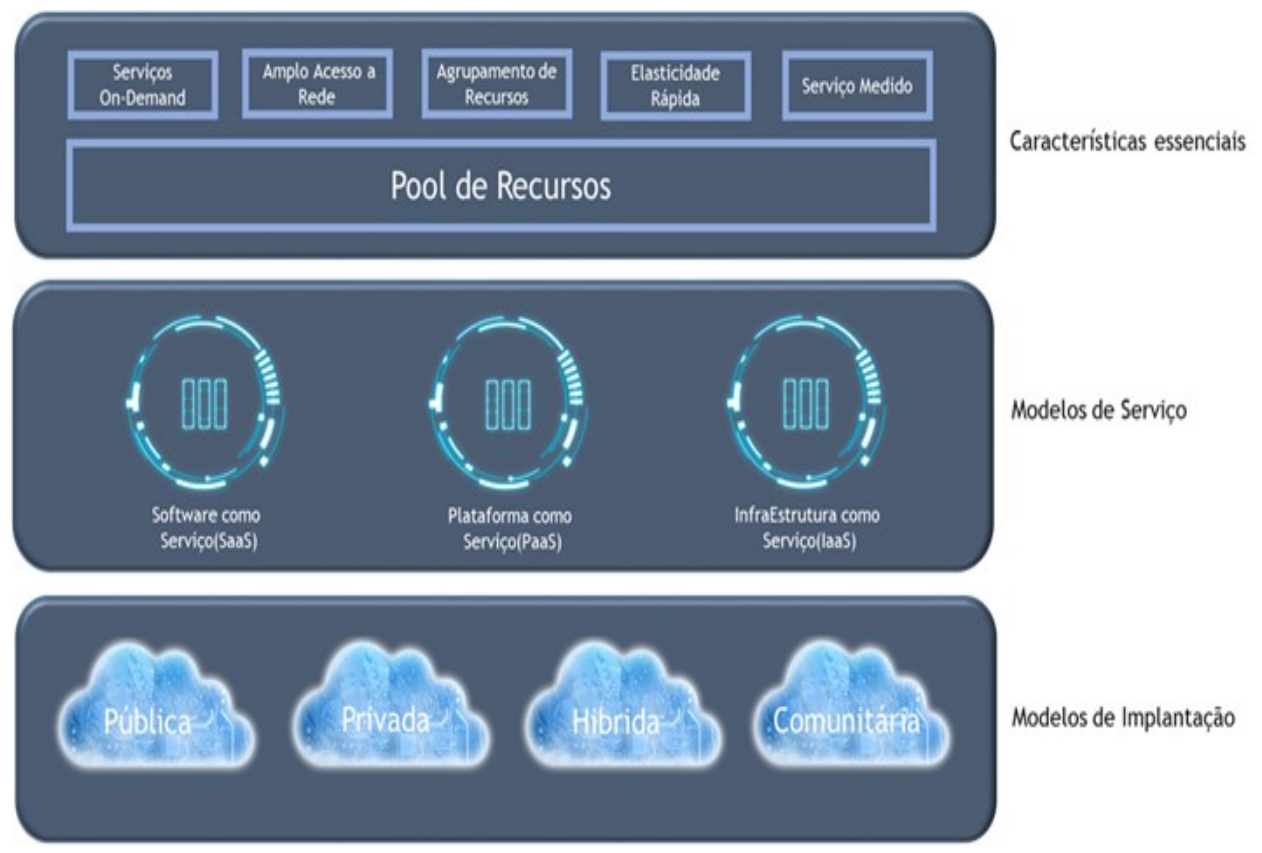

Fonte: Adaptado de NIST SP 800-145, "A NIST definition of cloud computing", http://csrc.nist.gov/publications/drafts/800-145/Draft-SP-800145_cloud-definition.pdf

De acordo com Mell (2009):

A computação em nuvem é um modelo de rede onipresente que permite o acesso, conveniente e on-demand, a um conjunto de recursos de computação compartilhados, configuráveis (por exemplo, redes, servidores, armazenamento, aplicativos e serviços) e que podem ser rapidamente provisionados e liberados com o mínimo esforço de gerenciamento ou a interação do provedor de serviços. Este modelo de nuvem é composto por cinco características essenciais, três modelos de serviços e quatro implantações modelos.

As cinco características são:

- Self-service on-demand;

- Amplo acesso à rede;

- Agrupamento de recursos;

- Elasticidade rápida;

- Serviço medido.

- Os três modelos de serviço são os seguintes:

- Software como Serviço (SaaS);

- Plataforma como um serviço (PaaS);

- Infra-estrutura como um Serviço (laaS).

Os quatro modelos de implantação são os descritos a seguir:

- Nuvens privadas; 
- Comunidade de nuvens;

- Nuvens públicas;

- Nuvens híbridas.

As vantagens da adoção de Cloud Computing pela indústria 4.0 são inúmeras, segundo o relatório FORESIGHT (2013), os destaques são os seguintes:

- Flexibilidade e fácil compartilhamento de dados: uma vez que a nuvem não é uma localização física, várias pessoas podem acessar os mesmos arquivos de vários locais, desde que sejam permitidos. No caso de uma empresa e/ou indústria, isso garante grande agilidade. Com a adoção da Cloud Computing, os funcionários podem interagir em tempo real com o conteúdo armazenado na nuvem tendo como consequências a colaboração e a integração entre os departamentos, permitindo uma comunicação rápida e fácil;

- Monitoramento de mobilidade: a Cloud Computing oferece melhor controle de operações e rastreamento rápido de requisitos. Mesmo que você não observe fisicamente. Isso permite uma melhor compreensão e tomada de decisão porque o gerente tem acesso a dados que podem monitorar de forma flexível e eficiente o desempenho de uma empresa e/ou indústria;

- Economia: com a introdução da Cloud Computing, uma indústria está ganhando rentabilidade. Isso se traduz na rentabilidade dos serviços de suporte (suporte e suporte técnico para problemas técnicos) e servidores. Além disso, o tempo de obtenção de informações é otimizado. Isso abre a possibilidade de acesso aos dados com maior flexibilidade, conforme comentado.

\section{VISÃO E CONCEITO SOBRE A INDÚSTRIA 4.0}

Segundo Lucke et al. (2008) existe um consenso básico entre muitos pesquisadores que as revoluções industriais exigem um período de desenvolvimento em longo prazo e que abrangem os quatro aspectos, considerados como visões futuras de manufatura de acordo com vários autores:

- Fábrica: como um dos principais componentes da Indústria 4.0, a fábrica do futuro, vai funcionar com um novo conceito de integração, onde nem todos os recursos de fabricação (sensores, smart grids, máquinas, robôs, esteiras, etc.) estão conectados e trocando informações de forma automática, entretanto ela deve ser dotada de recursos que a torne inteligente o suficiente para prever as suas necessidades futuras; para controlar a produção processar e gerenciar os sistemas fabris. Este tipo modelo de fábrica é conhecido atualmente com Smart Factory;

- O Negócio: a Indústria 4.0 adota um conceito de comunicação completa, existe a necessidade de uma rede de telecomunicações entre várias empresas, fábricas, fornecedores, logística, recursos, clientes, etc. Cada unidade otimiza sua configuração em tempo real, dependendo das 
demandas e status das unidades associadas na rede, as informações e recursos são compartilhados em tempo real;

- Produtos: Um dos benefícios agregados a Indústria 4.0 será o fornecimento de um novo tipo de produto, gerado a partir da fabricação, ou seja, a inteligência embarcada nos produtos. Esses produtos vão ser integrados com sensores, componentes identificáveis e processadores, carregam informações e conhecimento e os produtos transmitem funcionalidades e o feedback dos usos para os sistemas de fabricação, com essas funcionalidades, muitas funções podem ser adicionadas aos produtos. Além disso, o log de informações pode ser incorporado ao desenvolvimento produtos, auxiliando na otimização do projeto, na previsão de demanda e na manutenção;

- Clientes: os clientes terão muitas vantagens interagindo com a Indústria 4.0. Um novo método de compra será ofertado aos clientes, ele vai permitir aos clientes fazerem pedidos para qualquer tipo e produto e em qualquer quantidade, além disso, os clientes podem alterar os seus pedidos a qualquer momento durante a produção, mesmo no último minuto e sem nenhum custo, por outro lado, o benefício da inteligência dos produtos permite ao cliente não só conhecer as informações de produção, mas também receber instruções de utilização dependendo de seus próprios comportamentos e meios de uso.

Segundo Schwab (2016), as principais alterações esperadas na Indústria em geral são as seguintes:

- Ampliação das expectativas dos clientes;

- Produtos com inteligência embarcada e mais produtiva;

- Novas maneiras de colaborar e mais parcerias;

- A transformação do modelo operacional para o modelo digital.

A figura 2 ilustra as várias fases da evolução da indústria.

Figura 2-Fases da Evolução da Indústria

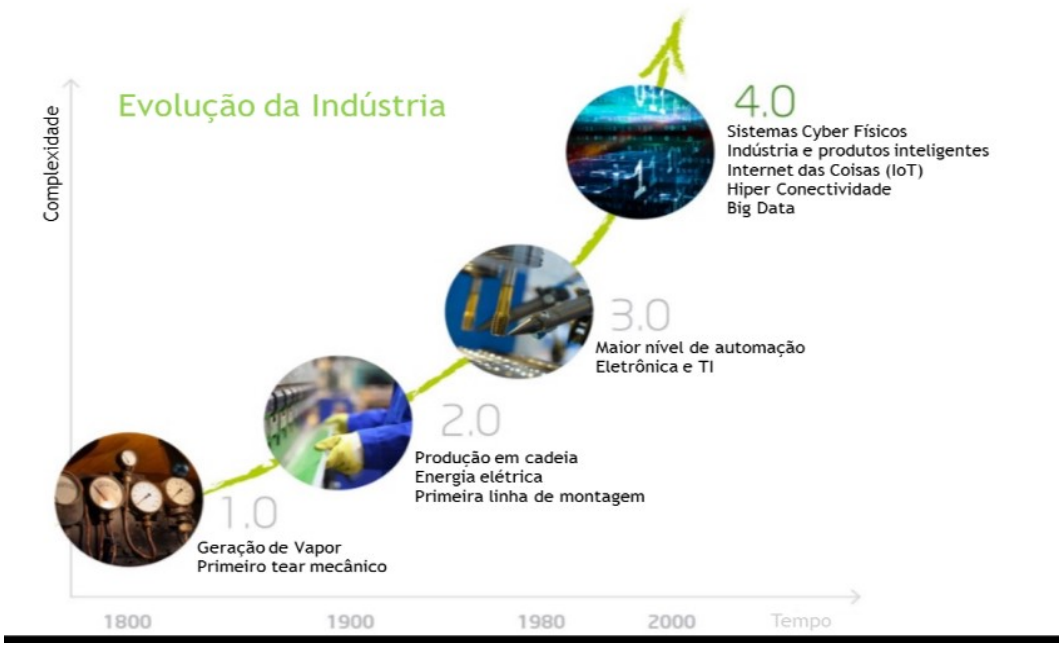

Adaptado com base na Zukunftsprojekt Industrie 4.0 


\section{DEFINIÇÃO SOBRE INTERNET OF THINGS (IOT)}

Na visão de Atzori et al. (2011), a loT é uma conexão estrita entre os mundos digitais e físicos. Inúmeros autores descrevem a loT de várias formas. Segundo Kranenburg (2008):

Uma infraestrutura de rede global dinâmica com capacidades de autoconfiguração baseadas em protocolos de comunicação padrão e interoperáveis, nos quais "coisas" físicas e virtuais têm identidades, atributos físicos e personalidades virtuais.

A loT pode ser definida como comunicação Machine to Machine (M2M) através da Internet, onde vários objetos, de automóveis a máquinas industriais ou bens de consumo, como roupas e calçados, podem compartilhar informações e dados para tarefas específicas. A base da operação da loT são sensores e devices que permitem a comunicação entre "coisas". Também requer um sistema computadorizado para analisar os dados recebidos e gerenciar as ações de cada objeto conectado a essa rede.

A loT pode ser aplicada em diferentes setores do nosso cotidiano, seja para otimizar as atividades da indústria ou no dia a dia das pessoas conforme alguns exemplos a seguir:

- Casa: um sistema de monitoramento através de termostato pode detectar as condições climáticas atuais e, tomando base a sua temperatura favorita, previamente cadastrada no dispositivo, ajustar a temperatura da casa o usuário se sinta confortável quando chegar em casa;

- Automóvel e Logística: cita-se como exemplo os automóveis que estacionam sozinhos e os protótipos de veículos auto dirigíveis. No que tange o setor logístico temos sensores que acompanham o desempenho dos veículos informando sobre manutenções preventivas e corretivas além da integração entre veículos e vias, permitindo que as entregas sejam feitas de forma mais rápida, inteligente e eficiente;

- Cidades Inteligentes: as cidades inteligentes (Smart Cities) só serão possíveis graças a loT. Lixeiras, vagas de estacionamento, sensores de alerta contra inundações, frota pública rastreada, dentre outros exemplos. Desta forma os administradores públicos podem economizar tempo e dinheiro, quanto mais objetos estiverem conectados, mais inteligentes é a administração da cidade e, portanto, melhora-se a qualidade de vida dos cidadãos.

A figura 3 mostra as cinco fases no desenvolvimento da Internet, sendo que a primeira fase começa com a conexão de dois computadores, depois houve a criação da World Wide Web que possibilitou a conexão de um grande número de computadores.

A Internet móvel foi criada de forma a conectar dispositivos móveis à Internet, posteriormente possibilitou a interação das pessoas através de redes sociais. $\mathrm{E}$, finalmente, ela caminha para a Internet das coisas conectando todas as coisas cotidianas à Internet. 
Figura 3: As cinco fases da evolução da Internet

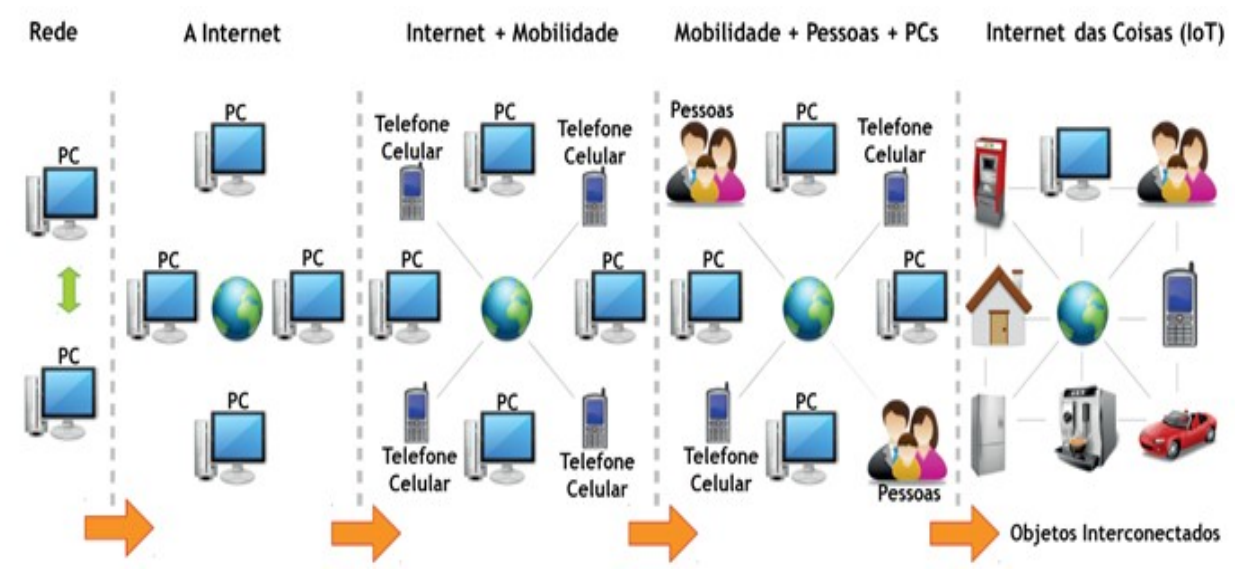

Fonte: Adaptado com base na figura disponível em:

htps://www.researchgate.net/publication/271585522_A_Survey_on_Internet_of_Things _From_Industrial_Market_Perspective

\section{DEFINIÇÃO SOBRE CYBER-PHYSICAL SYSTEMS (CPS)}

Segundo Rajhans et al. (2009), o termo Cyber-physical systems (CPS) refere-se a uma nova geração de sistemas com capacidades informáticas e físicas integradas que podem interagir com os seres humanos através de muitas novas modalidades.

A capacidade de interagir e desenvolver relações físicas através da computação, comunicação e controle é um fator chave nos futuros desenvolvimentos tecnológicos. As oportunidades de pesquisa e os desafios incluem o desenvolvimento e o desenvolvimento de aviões e veículos espaciais de próxima geração, veículos elétricos híbridos a gás, tráfego urbano totalmente autônomo e próteses que permitem controlar objetos físicos com sinais cerebrais.

A pesquisa sobre CPS visa integrar conhecimento e princípios de engenharia em disciplinas de informática e engenharia (rede, controle, software, interação humana, teoria da aprendizagem, bem como disciplinas de elétrica, mecânica, química, biomédica e materiais), além de desenvolver novas tecnologias para suporte ao Cyber-physical systems.

$\mathrm{Na}$ indústria, muitos sistemas de engenharia foram projetados desacoplando o design do sistema de controle dos detalhes da implementação de hardware / software. Na indústria automotiva, por exemplo, um sistema de controle de veículo depende dos componentes do sistema fabricados por diferentes fabricantes com seu próprio software e hardware.

Um grande desafio para fabricantes de equipamentos originais (OEMs) que fornecem peças para uma cadeia de suprimentos é reduzir custos, desenvolvendo componentes que podem ser integrados em diferentes veículos.

A crescente complexidade dos componentes e o uso de tecnologias avançadas para sensores e atuadores, comunicação sem fio e processadores 
multicore representam um grande desafio para os sistemas de controle de veículos da próxima geração.

Tanto o fornecedor como os integradores devem ser capazes de uma integração confiável e econômica de sistemas independentes.

Os componentes de hardware e software, middleware e sistemas operacionais devem ser desenvolvidos para ir além das tecnologias existentes atualmente, nestes casos os recursos computacionais requeridos só podem ser encontrados em larga escala em Cloud Computing. O hardware e o software devem ser altamente confiáveis, reconfiguráveis e, se necessário, certificáveis, componentes para sistemas totalmente integrados, esses sistemas complexos devem ter a disponibilidade e alto índice de confiabilidade que faltam em muitas infraestruturas computacionais instaladas on-site.

Alguns dos desafios no desenvolvimento dos Cyber-physical systems incluem:

- Algoritmos de otimização robótica em tempo real;

- Estruturas de otimização multi-stakeholder e multi-objetivo;

- Arquiteturas de sistemas distribuídos que facilitam a tomada de decisões;

- Fusão de dados com sensores heterogêneos para avaliação do valor da informação.

Espera-se que os Cyber-physical systems desempenhem um papel importante na concepção e desenvolvimento de futuros sistemas de engenharia com novas capacidades que excedem os níveis atuais de autonomia, funcionalidade, facilidade de uso, confiabilidade e segurança cibernética. A figura 4 mostra a interação dos Cyber-physical systems com dispositivos, máquinas, sensores e atuadores em integração total com a Internet.

Figura 4: Internet de coisas e Serviços

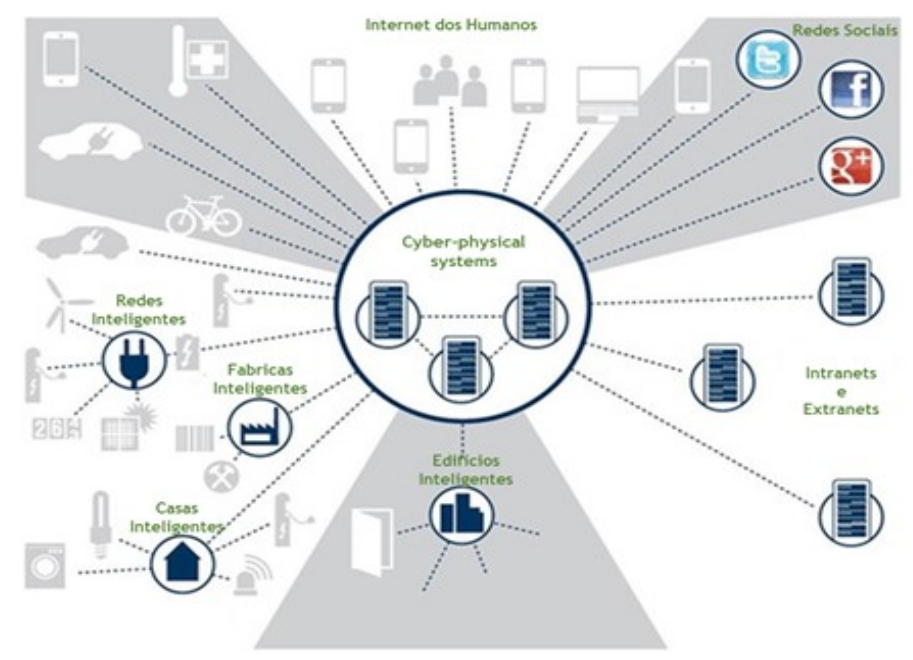

Fonte: Adaptado de Abschlussbericht des Arbeitskreises Industrie 4.0 


\section{PROCEDIMENTOS METODOLÓGICOS}

Com o mundo cada vez mais virtualizado, a Cloud Computing é considerada uma ferramenta importante para aumentar a produtividade, possibilitar uma conectividade eficaz entre unidades fabris e criar novas oportunidades para empresas de todos os tamanhos e segmentos.

As empresas estão sempre procurando novas maneiras para melhorar a produção e otimizar o tempo sem esquecer as melhorias na qualidade. As soluções de Cloud Computing oferecem capacidades de computação, armazenamento e rede sem precedentes. A tecnologia da Informação fornece plataformas para conectar a automação, a robótica e a loT, além de contribuir para desenvolvimentos inovadores a longo prazo.

O presente trabalho baseia-se em técnicas de pesquisa bibliográfica documental a livros, artigos, páginas de web sites, normas e dissertações.

De acordo com Mattar (2008), a pesquisa bibliográfica é, uma das maneiras mais rápidas e de menor custo, que podem clarear ou aprofundar um problema de pesquisa, através do conhecimento dos trabalhos realizados por outros, via levantamento bibliográfico.

Segundo Lakatos et al. (2001), a pesquisa bibliográfica tem como finalidade "colocar o pesquisador em contato direto com tudo aquilo que foi escrito sobre determinado assunto [...]".

Quanto ao objetivo, o estudo utiliza-se de pesquisa qualitativa onde foram utilizados dados estatísticos disponibilizados pela Confederação Nacional das Indústrias (CNI), Agência Brasileira de Desenvolvimento Industrial (ABDI), National Academy of Science and Engineering (ACATECH) e Policy Department A: Economic and Scientific Policy European Parliament.

Ao optar pela pesquisa bibliográfica, pensou-se na possibilidade de utilizarse das idéias de vários pesquisadores que conseguiram atingir seus objetivos e trazer acima de tudo, a escrita como objeto para pesquisas subseqüentes.

$\mathrm{O}$ artigo foi elaborado em um ambiente onde a autor carrega grande conhecimento no assunto abordado, o que facilitou na elaboração e na apresentação dos tópicos.

\section{APRESENTAÇÃO E DISCUSSÃO DOS RESULTADOS}

De acordo com Haug et al. (2016), a Cloud Computing é considerada uma inovação na arquitetura de computadores, e sua característica principal é a virtualização de recursos e serviços de computação. Ela permite que os recursos de computação sejam entregues com cinco características principais, ou seja, self-service on-demand, amplo acesso à rede, agrupamento de recursos, elasticidade rápida e serviço medido. Desta forma a Cloud Compunting é uma tecnologia chave para implementação da Indústria 4.0.

A tecnologia da informação está transformando os processos de fabricação de forma global digitalizando praticamente todas as etapas de fabricação, e esta 
característica é chamada de Indústria 4.0 na Europa e Smart Factory nos Estados Unidos.

Segundo Ezell (2016):

A Cloud Computing, em conjunto com outras tecnologias essenciais, tais como, sensores sem fio avançados, computação de alto desempenho, design assistido por computador (Computer-Aided Engineering - CAD), engenharia assistida por computador (Computer-Aided Engineering - CAE), manufatura assistida por computador (Computer-Aided Manufacturing - CAM), representam um componente essencial da revolução de fabricação inteligente.

A Cloud Computing vai interagir praticamente com todos os aspectos das empresas modernas no que tange os processos de fabricação. No nível das corporações, a computação em nuvem terá um impacto na forma como as empresas gerenciam suas operações, desde o planejamento de recursos empresariais (Enterprise Resource Planning - ERP), gerenciamento financeiro até a análise de dados além dos colaboradores.

A nuvem também integrará a cadeia de suprimentos de todos os fornecedores. Ao nível dos produtos, a Cloud Computing irá influenciar no projeto, desenvolvimento, na forma como eles são fabricados e como os clientes irão utilizá-los no dia a dia. Além desses aspectos, a computação em nuvem desempenhará um papel fundamental na validação e na universalização de novos sistemas de produção de produção, tais como os processos de fabricação utilizando impressão 3D e a loT.

As soluções baseadas na nuvem oferecem aos fabricantes uma ampla gama de benefícios, entre os mais importantes: escalabilidade; eficiência operacional; integração de aplicativos e parceiros; armazenamento, gerenciamento e análise de dados; e segurança aprimorada. Em particular, a computação em nuvem facilita a pesquisa, o design e o desenvolvimento de novos produtos, o que melhora a inovação, reduz os custos de desenvolvimento de produtos e aumenta a sobrevida destes produtos no mercado.

A Indústria 4.0 envolve a integração de tecnologias físicas e digitais, a integração das etapas de desenvolvimento, engenharia de produção e produção da cadeia para o uso final do produto e os serviços ligados a ela, além da operação de rede autônoma.

Representa muito mais do que a automação do processo industrial, por exemplo, a integração das máquinas e sistemas entre si (inclusive entre fábricas distintas de uma mesma cadeia de suprimentos), a conexão digital da máquina com o produto. Na figura 5 podem-se verificar as nove tecnologias que estão transformando a produção industrial, entre elas estão a Cloud Computing, a sendo um dos principais drivers da Indústria 4.0., pois provê armazenamento elástico e grande agrupamento de recursos computacionais.

Figura 5: Nove tecnologias que estão transformando a produção industrial. 


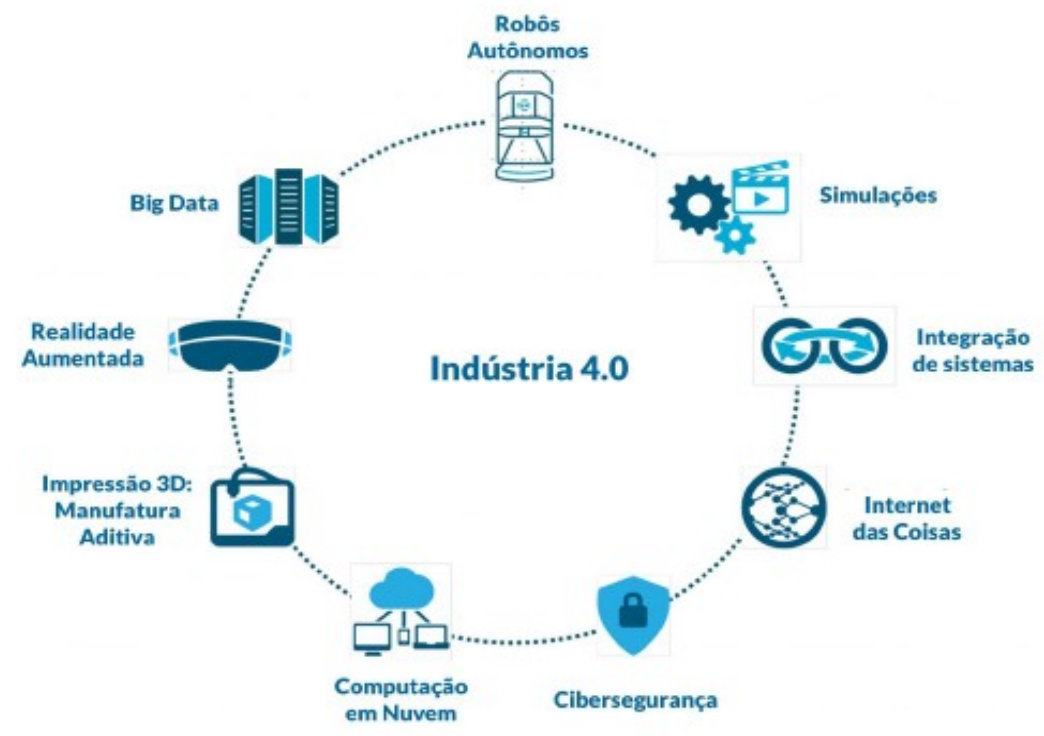

Fonte: https://www.slideshare.net/OpencaddAdvancedTech/apresentaodouglas-oliveira

\section{A CLOUD COMPUTING NO BRASIL}

Segundo o relatório "Sondagem 66" (2016) publicado em Confederação Nacional da Indústria (CNI), o uso de tecnologias digitais na indústria brasileira não é generalizado, pois no universo de todas as indústrias do Brasil apenas $58 \%$ conhecem a importância dessas tecnologias para a competitividade industrial e menos da metade as utiliza. $O$ foco das que utilizam é melhorar o processo de produção, aumentando a produtividade.

A pesquisa também abrangeu um grupo de 2225 empresas, de todos os portes, e nestas empresas $73 \%$ relataram usar pelo menos uma tecnologia digital em seus processos de fabricação, outros $47 \%$ disseram que confiaram nessas ferramentas para desenvolver a cadeia de produção e apenas $33 \%$ relatam usar sistemas digitais para gerenciar novos produtos e empresas. A CNI descobriu que quase a metade das empresas pesquisadas possuía pelo menos uma tecnologia digital baseada em pesquisa, como a automação digital sem sensores, prototipagem rápida ou impressão em 3D além de serviços na nuvem.

Segundo a CNI, os setores que estão mais avançados na adoção dos princípios e práticas da Indústria 4.0 são os de computadores, dispositivos eletrônicos e ópticos. A indústria de eletrônicos, comunicações e dispositivos médicos usa pelo menos uma das tecnologias listadas avaliadas pela CNI, com $61 \%$ de responsabilidade. O segundo lugar neste ranking é o segmento de máquinas e materiais elétricos, que representa $60 \%$ do uso de certas tecnologias nos processos de produção. 
De acordo com o relatório "Top Markets Report Cloud Computing", publicado pela International Trade Administration (2016), o Brasil ocupa a 4a posição e apresenta um mercado grande, sofisticado e crescente para uma variedade de serviços relacionados à nuvem.

Uma pesquisa da McKinsey's (2015), indica que as tecnologias disruptivas empregadas na Indústria 4.0, tais como, fabricação habilitada pela Tecnologia da Informação (TI) e o aumento da capacidade de computação, vão manter as Smart Factories altamente eficientes e com os processos cada vez mais integrados aos dados na nuvem.

Um dos objetivos deste relatório era definir as tecnologias disruptivas, habilitadas digitalmente, que provavelmente terão um impacto significativo na manufatura nos próximos 10 anos. Algumas dessas tecnologias são inovações reais, como a realidade aumentada, enquanto outras, como Big Data e Analitycs, já são aplicadas nas indústrias há algum tempo. A figura 6 mostra uma série de tecnologias disruptivas que permitirão a digitalização do setor de fabricação.

Figura 6: Digitalização do setor de fabricação - Indústria 4.0.

\begin{tabular}{|c|c|c|c|}
\hline $\begin{array}{l}\text { Big Data / Dados abertos } \\
\text { Custos significativamente } \\
\text { reduzidos de computação, } \\
\text { armazenamento e sensores } \\
\text { Internet das coisas / M2M } \\
\text { Custo reduzido do hardware de } \\
\text { pequena escala e } \\
\text { conectividade (por exemplo, } \\
\text { através de redes LPWA) } \\
\text { Cloud Computing } \\
\text { Centralização de dados e } \\
\text { virtualização do } \\
\text { armazenamento }\end{array}$ & $\begin{array}{l}\text { Digitalização e automação } \\
\text { do trabalho e do } \\
\text { conhecimento } \\
\text { Avanços em } \\
\text { inteligência artificial } \\
\text { e nas maquinas de } \\
\text { aprendizado } \\
\text { Análise avançada } \\
\text { Algoritmos aprimorados e } \\
\text { melhoria em grande } \\
\text { Escala na disponibilidade } \\
\text { de dados }\end{array}$ & $\begin{array}{l}\text { Interfaces de toque } \\
\text { e novas interfaces } \\
\text { de uso gráficas } \\
\text { Proliferação rápida através } \\
\text { de dispositivos de consumo } \\
\text { Realidade virtuale } \\
\text { aumentada } \\
\text { Avanço de displays óticos de } \\
\text { cabeça (por exemplo, Google } \\
\text { Glass) }\end{array}$ & $\begin{array}{l}\text { Fabricação de aditivos (isto é, } \\
\text { impressão em 3D) } \\
\text { Ampliação da gama de } \\
\text { materiais, em declínio rápido } \\
\text { preços para impressoras, } \\
\text { aumento precisão / qualidade } \\
\text { Robótica avançada (por } \\
\text { exemplo, colaboração entre } \\
\text { humanos e robôs) } \\
\text { Avanços em inteligência } \\
\text { artificial, visão de máquina, } \\
\text { comunicação M2M e atuadores } \\
\text { mais baratos } \\
\text { Armazenamento e captação } \\
\text { energia } \\
\text { Vão se tornar cada vez mais } \\
\text { rentável as opções para coletar } \\
\text { e armazenar energia }\end{array}$ \\
\hline
\end{tabular}

ronte: Aaаpтаao ae ntтps://www.mckınsey.ae/tוles/mck_ınaustry_4U_report.pat

\section{A INDÚSTRIA 4.0 NO BRASIL E EM ALGUNS PAÍSES DO MUNDO}

Segundo o relatório "Panorama da Inovação - Indústria 4.0" (2016) da Federação das Indústrias do Rio de Janeiro, a indústria brasileira ainda se encontra em grande parte na transição do que seria a Indústria 2.0, caracterizada pelo uso de linhas de montagem e energia elétrica, para a Indústria 3.0, que utiliza automação através do uso intenso da eletrônica, ativos robóticas e programação. 
A vertical que está mais preparada para assimilar as características de produção presentes na Indústria 4.0 é a automobilística.

A indústria automotiva possui profissionais altamente capacitados e que estão em processo constante de aperfeiçoamento, além de terem várias aplicações hospedadas na nuvem, tais como Eletronic Data Interchange (EDI) e aplicações de CAD/CAM. Outros setores da indústria, como óleo e gás e aeroespacial, possuem profissionais altamente qualificados e utiliza-se de muitas aplicações hospedadas dentro da arquitetura de Cloud Computing.

Em 2011, na feira de Hannover, a Alemanha, tentando recuperar a sua participação no valor agregado da indústria global, cunhou o termo "Industrie $4.0^{\prime \prime}$, e o novo conceito surgiu como parte da estratégia do governo para o desenvolvimento da manufatura do país utilizando-se de alta tecnologia.

Aumentar a produtividade através da inovação e elevar a competitividade com relação à manufatura de alguns países asiáticos eram alguns dos objetivos da estratégia do governo alemão.

Segundo a chanceler alemã Ângela Merkel: "A indústria 4.0 é a transformação completa de toda a esfera da produção industrial através da fusão da tecnologia digital e da internet com a indústria convencional".

De acordo com dados do relatório "Industry 4.0 The Future of Productivity and Growth in Manufacturing Industries" elaborado pelo Boston Consulting Group, estima-se que até 2025 ocorra o aumento da produtividade da indústria alemã podendo alcançar cifras de $€ 90$ bilhões a $€ 150$ bilhões, dependendo do setor industrial.

Projeta-se o aumento da receita em cerca de $€ 30$ bilhões por ano, o equivalente a $1 \%$ do PIB do país, já que a customização gerará maior demanda tanto por parte dos consumidores finais (bens manufaturados) quanto de equipamentos especializados. Aliado a tudo isso se projeta o aumento da necessidade de mão de obra qualificada, principalmente no setor de Engenharia Mecânica, desenvolvimento de software e Tecnologia da Informação (TI).

Segundo KURFUSS (2016) os Estados Unidos lançaram dois programas de incentivo a indústria, o Advanced Manufacturing Partership (AMP) e o National Network for Manufacturing Innovation (NNMI). O primeiro é um esforço do governo para promover a união da indústria, universidades e o governo federal visando o investimento em tecnologias emergentes de forma a criar mão de obra altamente qualificada para a indústria norte americana, aumentando a competitividade global dos insumos produzidos nos Estados Unidos.

O segundo consiste na criação de centros (HUBS) regionais com o objetivo de acelerar o desenvolvimento e a adoção de tecnologias de fabricação de ponta para fazer novos produtos globalmente competitivos.

De acordo com IAO (2016) a China anunciou em março de 2015 um projeto estratégico baseado no modelo alemão, sendo o objetivo atualizar a indústria chinesa de forma a torná-la mais eficiente e integrada, aumentando mais ainda a participação do país na cadeia de produção global.

O plano baseia-se nos seguintes aspectos principais: desenvolver não apenas indústrias avançadas, mas tradicionais, foco no sistema de normas internacionais, ao invés de normas técnicas nacionais, definição de medidas 
claras e específicas para inovação, qualidade, fabricação inteligente e produção verde. As metas foram estabelecidas para serem alcançadas entre 2020 e 2025.

Um dos projetos chineses denominados de Made in China 2025, propõe cinco projetos: construção de centros de inovação em manufatura, projetos de fabricação inteligente; projetos de fortalecimento da indústria de base; projetos de fabricação com tecnologia verde e projetos de inovações disruptivas em equipamentos.

De acordo com o relatório "Factories of the Future PPP" (2013), elaborado pela Comissão Europeia, foi lançado o Factories of The Future (FoF) Public-Private Partnership (PPP), que é uma iniciativa dos países que compõem a zona do Euro que visa ajudar as indústrias do bloco a se adaptarem aos desafios competitivos globais. Um dos objetivos da ação é aumentar o uso de tecnologias facilitadoras em diversos setores de forma a ampliar a base tecnológica industrial através do desenvolvimento e integração de novas tecnologias para o uso da tecnologia da informação na manufatura e manuseio industrial de materiais avançados.

\section{ALGUMAS NECESSIDADES DA INDÚSTRIA 4.0 QUE SÃO SUPRIDAS PELA CLOUD COMPUTING}

A revolução industrial trouxe grandes mudanças aos mercados no decorrer do tempo, com a chegada da energia elétrica houve a introdução de um grande número de máquinas que permitiram a expansão da atividade industrial ao longo dos séculos XVIII e XIX.

Com o desenvolvimento dos processos produtivos a capacidade de produção atingiu um nível muito alto naquela época, iniciando-se a produção em massa, o que estimulou os consumidores em busca de mais opções de consumo.

A "Capacidade de Expansão" é um dos itens primordiais das indústrias, e esta premissa sempre foi necessária para atender à crescente demanda do mercado. Neste aspecto a presença de um grande número de máquinas foi visto durante bastante tempo como capacidade de produção instalada.

Nos dias de hoje, com a introdução do Cloud Compuntig não é mais assim, na realidade quanto menor for o número de máquinas e parques desatualizados melhor para a produção e o negócio. Com o parque de máquinas atualizado, existe a necessidade do uso crescente de recursos computacionais avançados que muitas vezes as empresas não têm condição de investir. A nuvem permite o acesso a estes recursos sem que haja investimento na aquisição, e ela também permite que aconteça a expansão desses recursos de forma on-demand e com grande elasticidade.

Armazene e expanda recursos de infraestrutura de acordo com as necessidades do negócio, as soluções de Cloud Computing foram criadas para permitir que se aumente ou diminua as suas capacidades de acordo com suas necessidades sem ter que disponibilizar recursos. A otimização desses investimentos permitirá a expansão das empresas no seu core business, ou seja, nas áreas mais estratégicas.

As empresas no cenário da Indústria 4.0 necessitam tomar decisões rapidamente e devem estar preparadas paras as mudanças que o mercado 
globalizado apresenta a cada hora, além disso, as inúmeras inovações que este modelo proporciona afeta, também, o público consumidor, que em função das ofertas e possibilidades de alterar o produto levam a criação de novas demandas a cada hora.

O "Monitoramento" on-line ajuda as empresas a identificar estas demandas e permite análise da performance de sua infraestrutura para que atenda de forma esperada a estas demandas instantâneas.

As soluções de Coud Computing permitem que estas empresas tenham um controle maior das suas operações, inserindo neste controle o diferencial da mobilidade, fazendo com que os gestores acompanhem o andamento das demandas e atuem em tempo real. Caso necessário, de qualquer local que eles se encontrem utilizando uma variada gama de plataformas computacionais, tais como, IPhones, SmartPhones, IPADS, Tablets, Notebooks, etc.

A indústria 4.0 é fortemente apoiada na "Automação de Funções" e uma das vantagens do uso da Cloud Computing é permitir que os sistemas computacionais presentes em várias plataformas de produção que controlam os processos de automação possam rodar em um ambiente multiplataforma, garantindo a uniformidade de procedimentos.

Existe uma busca incessante por parte da indústria na busca de novas maneiras de agilizar a produção sem deixar de lado a qualidade. A situação ideal é aquela onde se eleva a produção, mantendo níveis de controle cada vez apurados, de forma a evitar falhas, interrupções, descontinuidade ou desvio do padrão.

As soluções em Cloud Computing garantem "Agilidade" e desempenho, pois permitem o crescimento das ferramentas de colaboração integrando-as entre os vários setores da empresa. Esta agilidade na integração permite uma produção mais rápida e uma comunicação uniforme, e estes fatos reduzem e minimizam os erros. Outra vantagem proporcionada pela agilidade que a nuvem disponibiliza diz respeito aos casos de Disaster Recovery, ou seja, em caso de paradas nos sistemas de ERP, CAD, CAM, além de outros, a recuperação em um ambiente de nuvem é extremamente rápida e confiável.

\section{CONCLUSÕES}

O verdadeiro potencial do uso da Cloud Computing, como tecnologia de apoio a quarta revolução industrial, só pode ser alcançado através da integração das plantas fabris com as plataformas de TI na nuvem.

A Indústria 4.0 está avançando graças a evolução loT, dos Cyber-physical systems, da automação, da robótica e do uso intensivo da Cloud Computing. Essas tecnologias, utilizadas em consonância, com Big Data e Analytics, são elementos chave desta nova revolução industrial, comumente chamada de Indústria 4.0.

A utilização em grande escala da Cloud Computing está impulsionando a evolução de uma variedade de setores. Na manufatura, plantas inteligentes são instaladas obtendo-se melhorias significativas na eficiência e produtividade. No setor de serviços financeiros, a automação é usada para processar um volume 
crescente de dados, seja para atendimento ao cliente ou para se concentrar em áreas como segurança e risco.

A tecnologia da nuvem é um elemento chave da próxima revolução industrial, proporcionando às empresas os meios para inovar em torno dessas tecnologias. Independentemente do setor, a Cloud Compuntig é uma tecnologia crítica para o sucesso da próxima revolução industrial, pois ela fornecerá os meios para as empresas inovarem em torno de tecnologias disruptivas, a utilização maciça de laaS (Infrastructure as a Service), que é uma infraestrutura de computação instantânea, provisionada e gerenciada pela Internet que proporciona o aumento ou a redução da demanda de forma automática, o uso da PaaS (Platform as a Service), que consiste na hospedagem e implementação de hardware e software, via internet, e que é utilizada para prover aplicações SaaS (Software as a Service).

É importante frisar que os serviços na nuvem vão suportar os volumes de trabalho de todos os conjuntos de dispositivos das plantas industriais e que são cada vez mais diversos na Indústria 4.0.

O serviço na nuvem permite-nos dimensionar novos aplicativos, desenvolvidos de forma inovadora, utilizando-se de uma plataforma de nuvem integrada, e isso pode ser feito de forma on-demand, com amplo acesso à rede, utilizando-se de um agrupamento de recursos, com rápida elasticidade e pagando pelo serviço medido, ou seja, pelo uso.

$\mathrm{Na}$ quarta revolução industrial, da qual estamos testemunhando o seu nascimento, a Cloud Computing proporcionará os meios para que as empresas de médio e grande porte possam competir através da inovação. A quarta revolução industrial já está presente em nossos dias, e como nas três anteriores, será conduzida pela integração de recursos na nuvem aliadas a nova tecnologia. 


\title{
The importance of cloud computing for industry 4.0
}

\begin{abstract}
The search for better productivity and quality indexes is increasing, as companies seek to find alternatives to be more competitive and survive in the globalized world. In this context, this article proposes to present the importance of cloud computing for Industry 4.0. The industry is currently undergoing a transformation aimed at the digitization of its manufacturing plants, as well as the implementation of intelligent concepts in manufacturing processes. Cloud computing, considered one of the pillars of the so-called fourth industrial revolution, directly assists in the digitization of production, creating new business models based on data made available in the cloud. With this information, users can predict the closure of some production lines, remote programming of supplementary lines, and make adjustments in real time. The use of cloud computing enables access to virtually unlimited computing resources, providing internet-based services of things that allow the interconnection of industrial equipment and robots, through sophisticated sensors that generate hundreds of thousands of information per second, this stored information and processed with extreme speed, allows to reach new levels of efficiency, productivity and quality. This study intends to show how cloud computing is of fundamental importance for industry 4.0, because with the correct use of its tools the manufacturing units become more agile and adaptable to the production variations, avoiding losses and reworking.
\end{abstract}

KEYWORDS: Cloud computing. Industry 4.0. IoT. 


\section{REFERÊNCIAS}

ACATECH. Umsetzungsempfehlungen für das Zukunftsprojekt Industrie 4.0 Disponível em: <https: // www.bmbf.de/ files/Umsetzungsempfehlungen_Industrie4_0.pdf>. Acesso em 08 nov. 2017.

ATZORI, Luigi; IERA, Antonio; MORABITO, Giacomo. The internet of things: a survey. Computer Networks, 2010. crossref

BCG. Industry 4.0 The Future of Productivity and Growth in Manufacturing Industries. Disponível em: <https:// www.bcg.com / publications /2015 / engineered_products_project_business_industry_4_future_productivity_growth_manu facturing_industries.aspx>.

CNI. Sondagem especial - Indústria 4.0. Disponível em:

<http://www.portaldaindustria.com.br/relacoesdotrabalho/media/publicacao/chamad as/SondEspecial_Industria4.0_Abril2016.pdf>. Acesso em 09 nov. 2017

COMMISSION, EUROPEAN. Factories of the Future PPP: towards competitive EU manufacturing. Disponível em: <http: // ec.europa.eu /research / press/ 2013/ pdf/ ppp /fof_factsheet.pdf>. Acesso em 27 nov. 2017.

EZELL, Stephen J. A Policymaker's Guide to Smart Manufacturing (Information Technology and Innovation Foundation, (2016).

FIRJAN. Industria 4.0. Disponível em: < http: // www.firjan.com.br /publicações /publicacoes-de-inovacao/ industria-4-0-1.htm>. Acesso em 05 dez. 2017

FORESIGHT. The Future Of Manufacturing: A New Era Of Opportunity And Challenge For The UK Project Report. Disponível em: http://hssmi.org/portfolio-items/foresightfuture-manufacturing-new-era-opportunity-challenge-uk-project-report/>. Acesso em 25 nov. 2017.

HAUG, K.Candel; Tobias Kretschmer; Strobel Thomas. Cloud Adaptiveness Within Industry Sectors-Measurement and Observations. Disponível em: http://www.sciencedirect.com/science/article/pii/S0308596115001238>. Acesso em 21 nov. 2017.

IAO, FRAUNHOFER. Industry 4.0: China moves into the fast lane. Disponível em: $<$ https://www.iao.fraunhofer.de/lang-en/about-us/press-and-media/1218-industry-4-0china-moves-into-the-fast-lane.html>. Acesso em 27 nov. 2017.

INTERNATIONAL,T Administration. 2016 Top Markets Report Cloud Computing. Disponível em: < https:// www.trade.gov/ topmarkets/ pdf/ Cloud_Computing_Top_Markets_Report.pdf>. Acesso em 05 dez. 2017.

KRANENBURM R. The Internet of Things. Disponível em: https://www.hiig.de/en/symp/4866-2/>. Acesso em 23 nov. 2017. 
KURFUSS, T. Office of Science and Technologiy Austria Washington, DC. Industry 4.0: Manufacturing in the United States. Disponível em: <http://ostaustria.org/bridgesmagazine/item/8310-industry-4-0>. Acesso em 25 nov. 2017.

LAKATOS, Eva Maria; MARCONI, Marina de Andrade. Metodologia Científica. São Paulo: Atlas, 2008.

LEE, Jay; Hung-An, Kao; Behrad, Bagheri. A cyber-physical systems architecture for industry 4.0-based manufacturing systems. Disponível em: https://pdfs.semanticscholar.org/8abf/04223c6162d07d3d02be48478aa7e5aa82bb.pdf >. Acesso em 01 nov. 2017

LUCKE D; Constantinescu, C; Westkämper, E. Smart Factory - A Step towards the Next Generation of Manufacturing. Disponível em: $<$ https: //

link.springer.com/chapter/10.1007/978-1-84800-267-8_23>. Acesso em 03 nov. 2017.

MCKINSEY. Industry 4.0 - How to navigate digitization of the manufacturing sector. Disponível em: <https://www.mckinsey.de/files/mck_industry_40_report.pdf >. Acesso em 05 dez. 2017.

OLIVEIRA, D. Sociedade 5.0. Disponível em: < https: //www.slideshare.net /OpencaddAdvancedTech/apresentao-douglas-oliveira>. Acesso em 09 nov. 2017

RAJHANS A; S.W. Cheng; B. Schmerl; B.H. Krogh; C. Aghi, and A. Bhave. An Architectural Approach to the Design and Analysis of Cyber-Physical Systems. Disponivel em: https://journal.ub.tu-berlin.de/eceasst/article/view/286>. Acesso em 03 nov. 2017.

SCHWAB, Klaus. The Fourth Industrial Revolution. Disponível em:

http://digitalcommons.osgoode.yorku.ca/cgi/viewcontent.cgi?article $=1023 \&$ context=th r. Acesso em 05 dez. 2017.

WAHLSTER, Wolfgang. From Industry 1.0 to Industry 4.0: Towards the 4th Industrial Revolution. Disponível em: <http:// www.dfki.de/wwdata/ Vortrag_SGAICO_Zuerich_27_05_13/Industry_4_0_The_Semantic_Product_Memory_as _a_Basis_for_Cyber-Physical_Production_Systems.pdf>. Acesso em 01 nov. 2017.

Recebido: 07 Jan. 2019

Aprovado: 10 Out. 2020

DOI: 10.3895/gi.v16n2.9317

Como citar:

PAZ, A.C. et al. A importância da computação em nuvem para a indústria 4.0. R. Gest. Industr., Ponta

Grossa, v. 16, n. 2, p. 166-185, Abr./Jun. 2020. Disponível em: https://periodicos.utfpr.edu.br/revistagi Correspondência:

Antonio Carlos Menezes Paz

Centro Universitário Farias Brito (FBUNI), Fortaleza, CE, Brasil.

Direito autoral: Este artigo está licenciado sob os termos da Licença Creative Commons-Atribuição 4.0 Internacional.

\section{(c) (1)}

